ARTICLE

\title{
Suppressed decomposition of organometal halide perovskites by impermeable electron-extraction layers in inverted solar cells
}

\author{
K.O. Brinkmann', J. Zhao ${ }^{1,2}$, N. Pourdavoud ${ }^{1}$, T. Becker1, T. Hu,3, S. Olthof ${ }^{4}$, K. Meerholz ${ }^{4}$, L. Hoffmann ${ }^{1}$, \\ T. Gahlmann', R. Heiderhoff', M.F. Oszajca ${ }^{5}$, N.A. Luechinger ${ }^{5}$, D. Rogalla ${ }^{6}$, Y. Chen $^{3}$, B. Cheng ${ }^{2} \&$ T. Riedl ${ }^{1}$
}

The area of thin-film photovoltaics has been overwhelmed by organometal halide perovskites. Unfortunately, serious stability concerns arise with perovskite solar cells. For example, methyl-ammonium lead iodide is known to decompose in the presence of water and, more severely, even under inert conditions at elevated temperatures. Here, we demonstrate inverted perovskite solar cells, in which the decomposition of the perovskite is significantly mitigated even at elevated temperatures. Specifically, we introduce a bilayered electronextraction interlayer consisting of aluminium-doped zinc oxide and tin oxide. We evidence tin oxide grown by atomic layer deposition does form an outstandingly dense gas permeation barrier that effectively hinders the ingress of moisture towards the perovskite and-more importantly-it prevents the egress of decomposition products of the perovskite. Thereby, the overall decomposition of the perovskite is significantly suppressed, leading to an outstanding device stability.

\footnotetext{
${ }^{1}$ Institute of Electronic Devices, University of Wuppertal, Rainer-Gruenter-Str 21, 42119 Wuppertal, Germany. ${ }^{2}$ College of Materials Science and Engineering, Nanchang University, 999 Xuefu Avenue, Nanchang 330031, China. ${ }^{3}$ College of Chemistry/Institute of Polymers, Nanchang University, 999 Xuefu Avenue, Nanchang 330031, China. ${ }^{4}$ Department of Chemistry, University of Cologne, Luxemburger Straße 116, 50939 Cologne, Germany. ${ }^{5}$ Nanograde AG, Laubisrütistrasse 50, 8712 Stäfa, Switzerland. ${ }^{6}$ Ruhr-Universität Bochum, RUBION, Universitätsstr. 150, 44801 Bochum, Germany. Correspondence and requests for materials should be addressed to T.R. (email: t.riedl@uni-wuppertal.de).
} 
S olar cells based on organolead halide perovskites have seen tremendous progress over the past 5 years ${ }^{1}$. Although their power conversion efficiency (PCE) has skyrocketed to reach a level of $>20 \%$ (ref. 2), concerns about their stability are still intimately linked to this technology ${ }^{3-5}$.

Generally, there is consensus that perovskites like $\mathrm{CH}_{3} \mathrm{NH}_{3} \mathrm{PbI}_{3}$ decompose to their constituents, that is, $\mathrm{HI}, \mathrm{CH}_{3} \mathrm{NH}_{2}$ and $\mathrm{PbI}_{2}$, in the presence of water ${ }^{6}$. Moreover, the intrinsic decomposition of $\mathrm{CH}_{3} \mathrm{NH}_{3} \mathrm{PbI}_{3}$ to $\mathrm{CH}_{3} \mathrm{NH}_{3} \mathrm{I}$ and $\mathrm{PbI}_{2}$, which is thermally activated and which occurs even under inert conditions, states a more fundamental problem ${ }^{7,8}$. At the same time, there are secondary effects of perovskite decomposition such as the degradation of functional building blocks in the solar cell because of the corrosive effect of the perovskite decomposition products ${ }^{9}$. Specifically, the corrosion of metal electrodes like $\mathrm{Ag}$ or $\mathrm{Al}$ has been identified to be a critical issue ${ }^{10}$. Overall, without proper concepts to overcome these reliability issues, the prospects of wide-spread application and commercialization of organolead halide perovskite technology may be significantly compromised.

Among the various device architectures for perovskite solar cells, the inverted planar geometry has been shown to yield devices essentially free of hysteresis ${ }^{11,12}$. Here, the photogenerated holes are extracted via the bottom electrode using a hole-extraction interlayer (for example, poly(3,4-ethylenedioxythiophene) polystyrene sulfonate (PEDOT:PSS)), while the electron extraction proceeds via the top electrode. The energetic alignment of $\mathrm{CH}_{3} \mathrm{NH}_{3} \mathrm{PbI}_{3}$ and $\left[6,6^{\prime}\right]$-phenylC61-butyric acid methyl ester (PCBM) has been shown to be especially favourable for the extraction of electrons from the conduction band of the perovskite ${ }^{13}$. To overcome the stability issues discussed above, several groups have introduced inverted planar perovskite cells based on metal oxides as electronand hole-extraction interfacial layers adjacent to the cathode and anode, respectively ${ }^{14,15}$. Chen et al. ${ }^{15}$ employed a sol-gel $\mathrm{Ti}(\mathrm{Nb}) \mathrm{O}_{x}$ electron-extraction layer that was aimed to protect the perovskite against moisture from ambient atmosphere. Because of its poor electrical conductivity of $10^{-5} \mathrm{~S} \mathrm{~cm}^{-1}$, the authors had to limit the thickness of the sol-gel $\mathrm{Ti}(\mathrm{Nb}) \mathrm{O}_{x}$ to $10 \mathrm{~nm}$. Moreover, it is known that sol-gel-derived layers state only relatively poor permeation barriers ${ }^{16}$. As a result, the device stability tests that afforded a lifetime of $1,000 \mathrm{~h}$ were done with additional encapsulation. Kaltenbrunner et al. ${ }^{17}$ have used $\mathrm{Cr}_{2} \mathrm{O}_{3} / \mathrm{Cr}$ at the cathode side to protect the metal cathode and thereby to improve the stability under ambient conditions, but still the efficiency was found to drop to $80 \%$ of its initial value after only $28 \mathrm{~h}$. Very recently, Guerrero et al. ${ }^{18}$ confirmed the protective effect of the $\mathrm{Cr}_{2} \mathrm{O}_{3} / \mathrm{Cr}$ interlayer but still found some severe degradation after only a few hours. You et al. ${ }^{14}$ have shown that devices based on indium tin oxide (ITO)/ $\mathrm{NiO} /$ perovskite/ $\mathrm{ZnO} / \mathrm{Al}$ were stable for tens of days in ambient conditions, whereas a reference device based on ITO/PEDOT:PSS/perovskite/PCBM/Al showed severe degradation within half a day. Especially, the $\mathrm{ZnO}$ layer, which has been spin coated from a nanoparticle dispersion on top of the perovskite, has been claimed to protect the $\mathrm{Al}$ electrode against corrosion. Very recently, Bush et al. ${ }^{19}$ have found that in their devices a similar nanoparticle-based $\mathrm{ZnO}$ layer next to the cathode did not provide any sufficient protection at elevated temperatures. As solar cells in outdoor conditions may reach a temperature significantly above $25^{\circ} \mathrm{C}$, the latter result appears to be extremely relevant. Other reports have evidenced rapid decomposition of $\mathrm{CH}_{3} \mathrm{NH}_{3} \mathrm{PbI}_{3}$ when in direct contact with $\mathrm{ZnO}^{20}$.

Here we propose an inverted cell architecture, where a bilayered aluminium-doped $\mathrm{ZnO}(\mathrm{AZO}) /$ tin oxide $\left(\mathrm{SnO}_{x}\right)$ electron-extraction layer (EEL) affords perovskite solar cells with a remarkable resilience against moisture and heat. $\mathrm{AZO}$ is excellently suited to extract electrons from the lowest unoccupied molecular orbital of fullerenes ${ }^{21}$. However, the nanoparticlebased AZO layer does not provide sufficient protection against the ingress of moisture or the egress of perovskite decomposition products. Thus, we add a thin $\mathrm{SnO}_{x}$ layer to form a bilayered $\mathrm{AZO} / \mathrm{SnO}_{x}$ EEL assembly. The $\mathrm{SnO}_{x}$ is grown by low-temperature atomic layer deposition (ALD) at $80^{\circ} \mathrm{C}$ that affords extremely dense, conformal and pinhole-free layers. Please note that there are reports where ALD layers have been directly deposited on top of perovskites to improve their resilience against ambient atmosphere and heat ${ }^{22}$. However, these ALD layers were not a functional part of a device, and therefore most of the experiments were based on electrical insulators like $\mathrm{Al}_{2} \mathrm{O}_{3}$. $\mathrm{SnO}_{x}$ layers grown by $\mathrm{ALD}$ are optically highly transparent, electrically conductive and provide outstanding gas permeation barrier properties with a water vapour transmission rate as low as $7 \times 10^{-5} \mathrm{~g}\left(\mathrm{~m}^{-2} \mathrm{day}^{-1}\right)$, even when grown at a low temperature ${ }^{23}$. Their water vapour transmission rate is orders of magnitude better than that of sputtered ITO thin films or that of solution processed metal oxide layers. The electrical conductivity of the $\mathrm{SnO}_{x}$ layer on the order of $5 \times 10^{-3} \mathrm{~S} \mathrm{~cm}^{-1}$ allows us to place it between the sensitive electrode and the photoactive layers without adding any noticeable series resistance. Thereby, the $\mathrm{SnO}_{x}$ provides outstanding protection of the perovskite against the ingress of moisture and, more importantly, at the same time it serves as permeation barrier against the out-diffusion of decomposition products of the perovskite. We will show that the sealing property of the $\mathrm{SnO}_{x}$ layer contains the decomposition products inside the cell and, thereby, the overall decomposition of the perovskite is significantly suppressed. Although cells based on $\mathrm{LiF} / \mathrm{Al}$ or AZO degrade within tens of hours in ambient air $\left(23^{\circ} \mathrm{C}\right.$ and $50 \%$ relative humidity $(\mathrm{rH})$ ), devices based on $\mathrm{AZO} / \mathrm{SnO}_{x}$ show superior stability of their solar cell characteristics even on a timescale of $>350 \mathrm{~h}$. More strikingly, at $60^{\circ} \mathrm{C}$ under inert atmosphere, AZO-based devices degrade within $100 \mathrm{~h}$ because of thermally induced decomposition of the perovskite. On the contrary, the suppressed out-diffusion of decomposition products because of the diffusion-barrier properties of the $\mathrm{AZO} / \mathrm{SnO} \mathrm{ELL}_{x}$ affords cells that are essentially unchanged even after $>1,000 \mathrm{~h}$ under the same conditions. Our paper reports a general strategy to achieve a substantially improved device lifetime in the case of photoactive materials that may come with concerns about their intrinsic compositional stability.

\section{Results}

Planar inverted cells. The devices used in this study are based on a planar inverted cell layout, where the holes are extracted via the bottom electrode (substrate electrode). The layer sequence is shown in Fig. 1. As hole-extraction layer, PEDOT:PSS is used, whereas for electron extraction a $100 \mathrm{~nm}$ thick PCBM layer is deposited on top of the typically $180 \mathrm{~nm}$ thick $\mathrm{CH}_{3} \mathrm{NH}_{3} \mathrm{PbI}_{3}$ perovskite layer. Directly adjacent to the PCBM we have used a $100 \mathrm{~nm}$ thick AZO layer prepared from a nanoparticle dispersion in isopropylalcohol. In some cells, a $\mathrm{SnO}_{x}$ layer is deposited on top of the AZO layer by ALD at $80^{\circ} \mathrm{C}$ to form the bilayered electron-extraction assembly $\left(\mathrm{AZO} / \mathrm{SnO}_{x}\right)$. The nominal thickness of the $\mathrm{SnO}_{x}$ layer is $20 \mathrm{~nm}$. As the AZO layer is derived from a nanoparticle dispersion, it contains pores that are partially coated with $\mathrm{SnO}_{x}$ because of the conformal nature of the ALD process. A more detailed discussion of the $\mathrm{SnO}_{x}$ growth on top of the AZO layer is provided in the Supplementary Fig. 1. Please note that we have also prepared devices with $\mathrm{SnO}_{x}$ deposited directly on top of PCBM, but the stability of the 
a

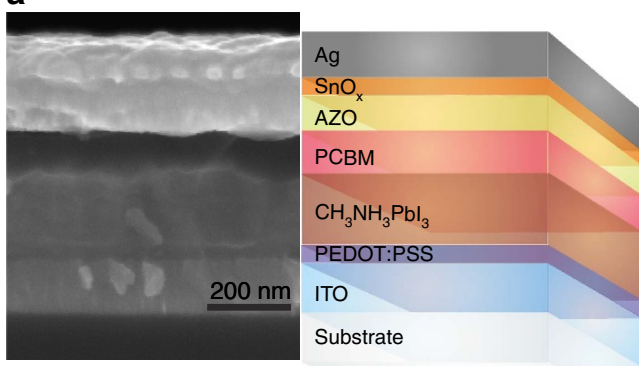

b

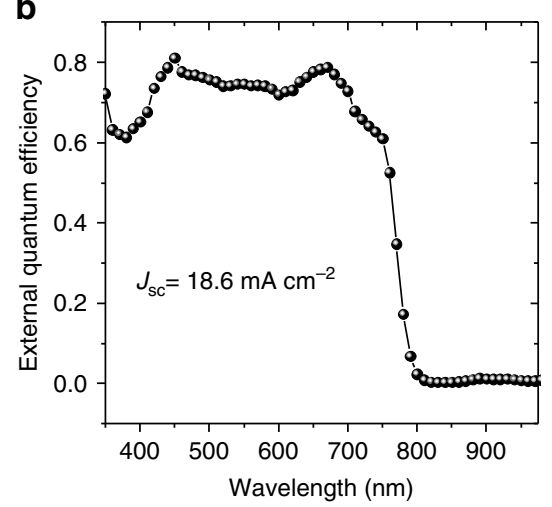

d

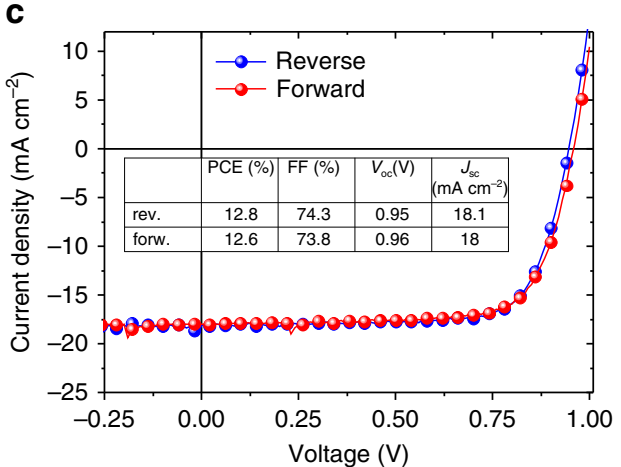

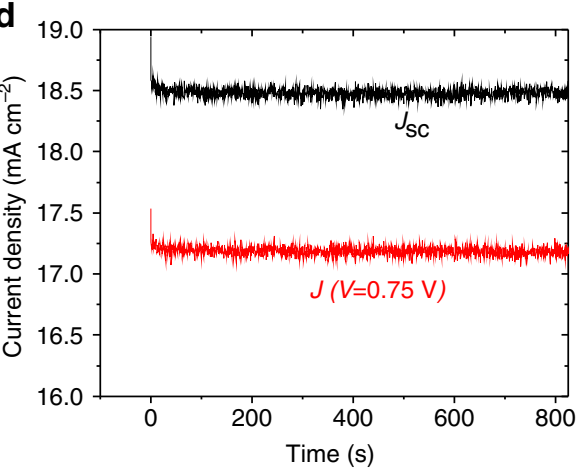

Figure 1 | Basic device characteristics. Scanning electron microscopy (SEM) image of the device cross-section along with the assignment of the respective layers (a). In some devices, the $\mathrm{SnO}_{x}$ or the AZO layer has been omitted. Note that the contrast between the $\mathrm{AZO}$ and the $\mathrm{SnO}$ layers in $\mathrm{SEM}$ is relatively poor. External quantum efficiency (EQE) of a representative device based on $\mathrm{AZO} / \mathrm{SnO}_{x}(\mathbf{b}) . J / \mathrm{V}$ characteristics measured in forward and reverse direction (c), and current density versus time under $0 \mathrm{~V}$ (black) and $0.75 \mathrm{~V}$ (red) bias (d).

resulting devices was inferior to that of the $\mathrm{AZO} / \mathrm{SnO}_{x}$ cells (see discussion below). Reference devices based on ITO/PEDOT:PSS/Perovskite/PCBM/LiF/Al have also been included in our study.

The external quantum efficiency (EQE) spectrum of a typical $\mathrm{AZO} / \mathrm{SnO}_{x}$-based perovskite solar cell is shown in Fig. 1b. The $J / V$ characteristics of the device measured in forward and reverse direction (scan speed: $500 \mathrm{mV} \mathrm{s}^{-1}$ ) are presented in Fig. 1c. No substantial hysteresis is found in the $J / V$ data. The extracted characteristics $V_{\mathrm{oc}}=0.95 \mathrm{~V}, \mathrm{FF}=74 \%$ and a $J_{\mathrm{sc}}=18 \mathrm{~mA} \mathrm{~cm}^{-2}$ result in a PCE of $12.8 \%$. We want to note that the perovskite precursor used in this study was commercially obtained from Ossila Ltd with a typically specified efficiency in the range of $11-13 \%$ (see Methods for details).

Significantly higher PCEs can be achieved by using, for example, optimized precursor inks or mixed-cation/mixedhalide perovskite absorbers ${ }^{24}$. As the EEL is in the core of this report, further optimization of the perovskite system itself was considered to be beyond the scope here.

Stability in ambient air. A very striking advantage of the bilayered $\mathrm{AZO} / \mathrm{SnO}_{x}$ EEL in direct comparison with $\mathrm{AZO}$ is displayed in Fig. 2. Here, the stability of the cell characteristics is shown for devices that were continuously exposed to ambient air at $23{ }^{\circ} \mathrm{C}$ and $50 \%$ relative humidity. The devices that were based on a single layer of AZO as EEL degrade swiftly within $\sim 50 \mathrm{~h}$. A severe decay of the FF from $\sim 70 \%$ to $<25 \%$ and a concomitant drop of the $J_{\mathrm{sc}}$ from $\sim 17$ to $8.2 \mathrm{~mA} \mathrm{~cm}^{-2}$ is found. Opposed to that, the characteristics of the corresponding cells with the bilayered EEL of $\mathrm{AZO} / \mathrm{SnO}_{x}$ do not show any degradation even after $>300 \mathrm{~h}$ in air. Note that the thickness of the $\mathrm{SnO}_{x}$ layer is only $20 \mathrm{~nm}$ in these devices. There is a slight increase of the $V_{\text {oc }}$ in the $\mathrm{AZO} / \mathrm{SnO}_{x}$ samples that happens on a timescale of several tens of hours. This increase is only observed in the $\mathrm{AZO} / \mathrm{SnO}_{x}$ samples, as the $\mathrm{LiF} / \mathrm{Al}$ and the AZO cells already undergo strong degradation on the same timescale. A similar increase of $V_{\mathrm{oc}}$ on such a long timescale has been seen by other authors and it has been explained by an ageing effect that reduces the density of trap states in the perovskite ${ }^{25}$. As a reference, the cells based on $\mathrm{LiF} / \mathrm{Al}$ are essentially degraded within $\sim 1$ day.

In order to analyse the reasons underlying the strikingly different degradation phenomena shown in Fig. 2, we have performed X-ray diffraction (XRD) and X-ray photoelectron spectroscopy (XPS) studies of the respective device structures. Note that in case of the XPS measurements, a thinner top Ag electrode was chosen $(10 \mathrm{~nm})$ compared with the actual solar cells $(100 \mathrm{~nm})$ because of the small probing depth of the technique. Looking first at the AZO-based device, the XRD spectrum shows the (110) and (220) reflections of tetragonal $\mathrm{MAPbI}_{3}$ at angles of $14.11^{\circ}$ and 28.14 , respectively. As this XRD measurement has been made on a full device stack, further signals related to the Ag and ITO electrodes are visible. A detailed assignment of these peaks is shown in the Supplementary Fig. 3. Only a very weak signal due to $\mathrm{PbI}_{2}$ was found that did not significantly increase even after 7 days in air (Fig. 3a). Thus, no severe decomposition of the perovskite to $\mathrm{PbI}_{2}$ upon storage in air can be inferred from the XRD data. On the other hand, when XPS was used to look for degradation products on the surface, a notable amount of iodine was evidenced on the AZO sample (Fig. 3b) after exposure to air for 2 days, whereas on identical samples kept in nitrogen no such 
a

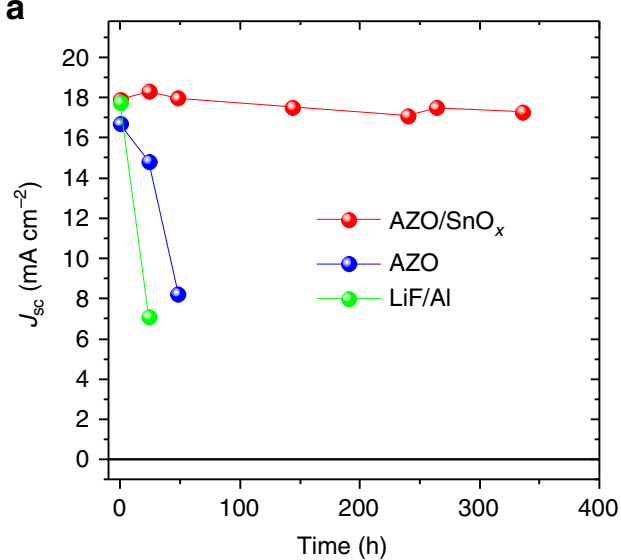

C

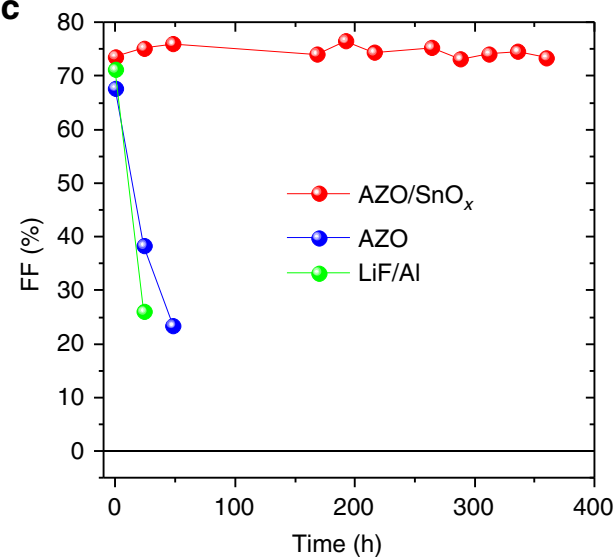

b

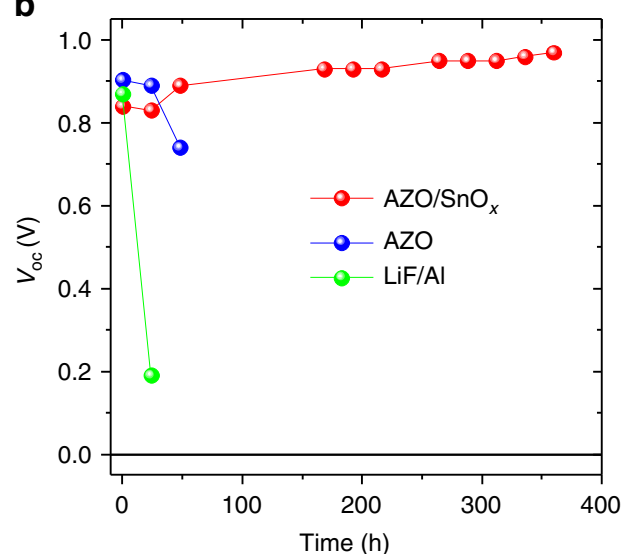

d

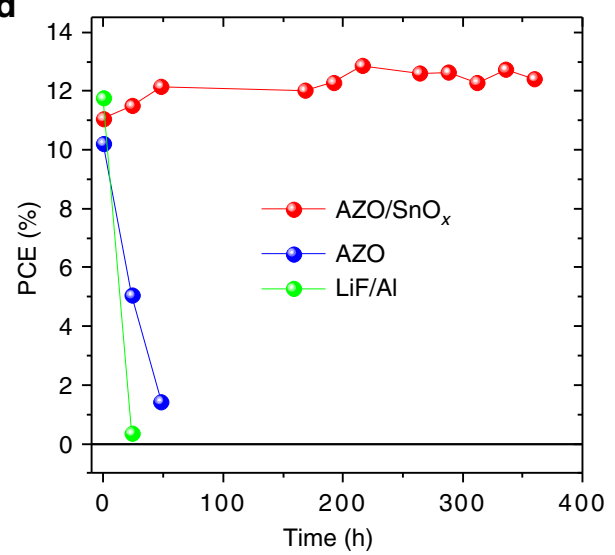

Figure 2 | Stability upon storage in ambient air. Characteristics of inverted perovskite collar cells with varied cathode/electron extraction assemblies $\left(\mathrm{AZO} / \mathrm{SnO}_{x} / \mathrm{Ag}, \mathrm{AZO} / \mathrm{Ag}\right.$ and $\mathrm{LiF} / \mathrm{Al}$ ) versus time of storage in ambient air (at $23^{\circ} \mathrm{C}$ and $\left.50 \% \mathrm{rH}\right) . \mathrm{J}_{\mathrm{sc}}(\mathbf{a}), \mathrm{V}_{\mathrm{oc}}(\mathbf{b}), \mathrm{FF}(\mathbf{c})$ and PCE (d). The characteristics have been determined from the $J / V$ measurements in reverse direction (examples in Supplementary Fig. 2).

iodine signal is found. Please note that the peak of $\sim 622 \mathrm{eV}$ in the XPS spectrum of the aged AZO cell does not represent another bonding state of the iodine, but it is rather a so-called shake-up peak. Such shake-up peaks are commonly observed in strong XPS signals. The survey XPS spectra can be found in the Supplementary Fig. 4. Plan-view scanning electron microscopy (SEM) images of the AZO samples aged in air show the formation of spot-like degradation motifs (Fig. 3c,d). These spots point to the local ingress of moisture and out-diffusion of decomposition products of the perovskite, supported by the appearance of the iodine signal in XPS. In the degraded spots, needle-like features occur. Similar features have been reported for Ag electrodes corroded by the decomposition products of $\mathrm{MAPbI}_{3}$ (ref. 26). Our findings are also in line with the results of Kato et al. ${ }^{10}$ who unveiled a moisture promoted corrosion mechanism of Ag which resulted in the formation of products like AgI. The role of water in the electrode corrosion is further clarified below in cells aged under inert conditions.

To explain the spot-like degradation, we can assume that structural defects, such as pinholes, in the AZO NP layer would give rise to locally enhanced water permeation. Opposed to that, the sample with the bilayered $\mathrm{EEL}$ of $\mathrm{AZO} / \mathrm{SnO}_{x}$ does not show any sign of degradation in SEM (Fig. 3e) under identical conditions. In XPS, only a negligible amount of iodine at the surface of the $\mathrm{AZO} / \mathrm{SnO}_{x}$ samples can be detected. For comparison, we have also studied layer stacks where only $\mathrm{SnO}_{x}$, that is, without AZO between the PCBM and the $\mathrm{SnO}_{x}$, has been used as EEL. We have recently shown that $\mathrm{SnO}_{x}$ with a work function of $4.1 \mathrm{eV}$ forms an excellent EEL layer in inverted organic solar cells ${ }^{27}$. Here, the resulting perovskite solar cells based on $\mathrm{SnO}_{x}$ were more stable than the devices based on AZO. However, they showed a statistical failure on a timescale of $100 \mathrm{~h}$ (Supplementary Fig. 5). This finding could be explained by a nonideal ALD growth of the $\mathrm{SnO}_{x}$ directly on top of the organic PCBM layer. ALD as a chemical deposition technique relies on self-limiting chemical reactions on the sample surface ${ }^{28}$. For the ALD deposition of oxide layers, $-\mathrm{OH}$ surface groups form the nucleation sites for layer growth. In this sense, on organic surfaces like PCBM the nucleation of an ALD layer may deviate significantly from the growth on a surface rich of -OH groups ${ }^{29}$. Therefore, we can assume that the $\mathrm{SnO}_{x}$ layer grown on PCBM is not free of some pin-hole defects that compromise its functionality as diffusion barrier. Consequently, substantially more iodine is detected on the surface after exposure to air compared with the $\mathrm{AZO} / \mathrm{SnO}_{x}$ device (Fig. 3b). Thus, we conclude that the AZO serves two purposes in the bilayered $\mathrm{AZO} / \mathrm{SnO}_{x}$ EEL: (1) its electronic structure has been shown to facilitate electron extraction from the PCBM (ref. 21) and (2) the AZO layer provides an improved (oxide-) surface for the nucleation of the subsequent $\mathrm{SnO}_{x}$ ALD layer that can thus from a dense, pin-hole-free permeation barrier. Figure $3 \mathrm{f}, \mathrm{g}$ shows schemes portraying the specific differences between AZO and $\mathrm{AZO} / \mathrm{SnO}_{x}$ as EEL in devices exposed to air.

As indicated before, the AZO layer prepared from the NP dispersion does not provide a significant diffusion barrier against the local ingress of moisture from the ambient air. 
a

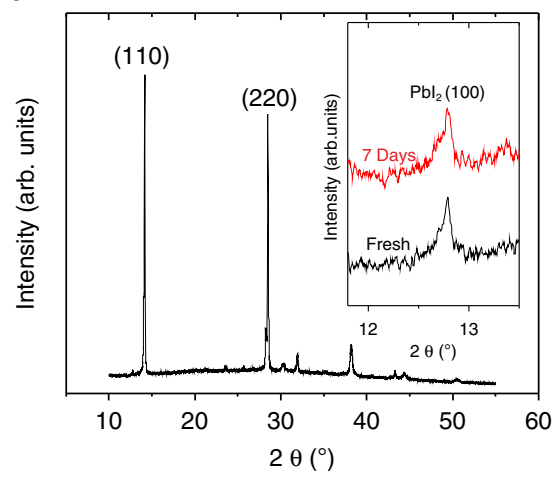

b

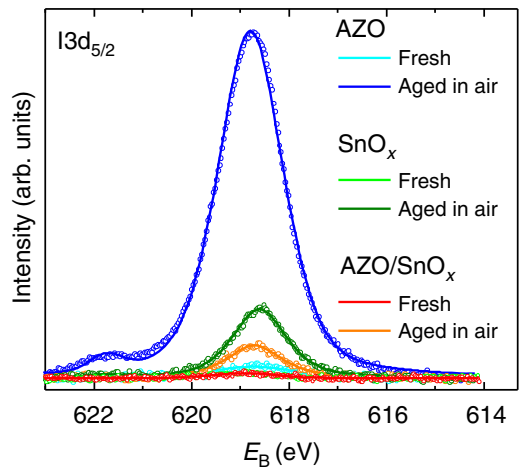

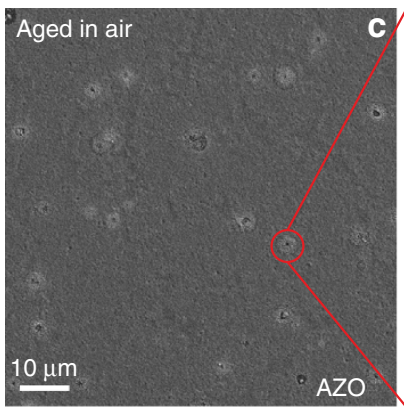
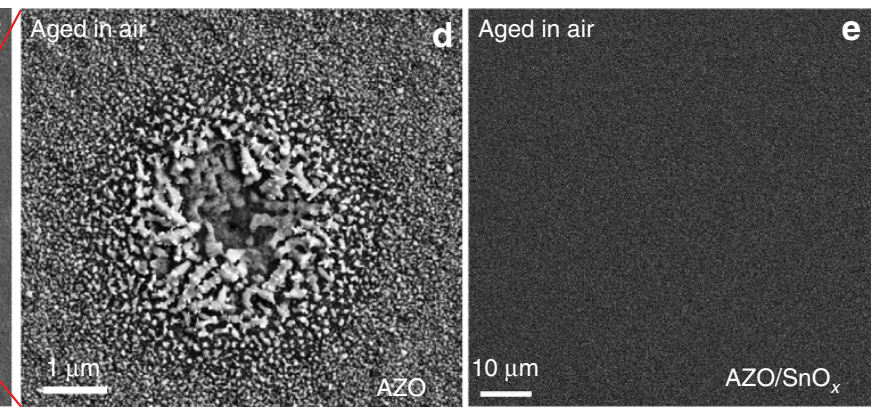

f
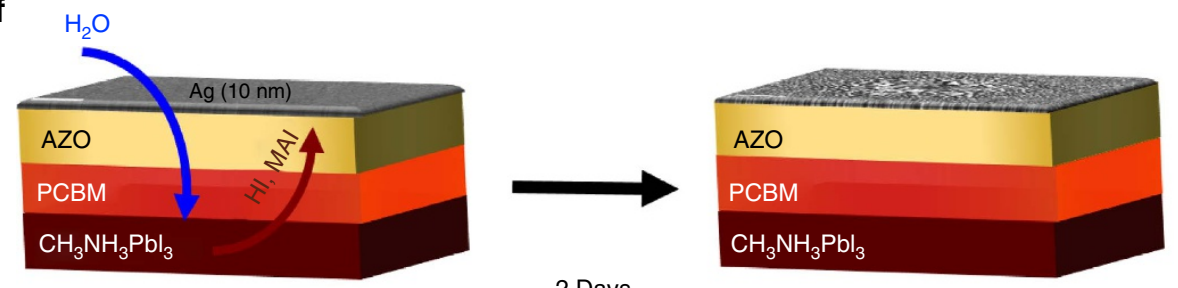

2 Days

g

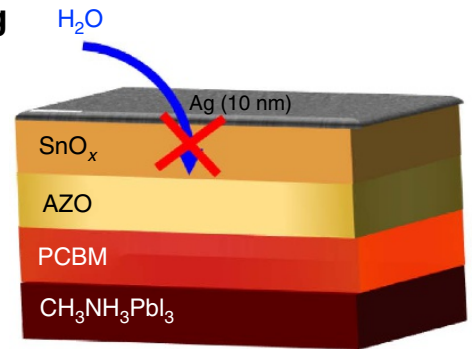

Air

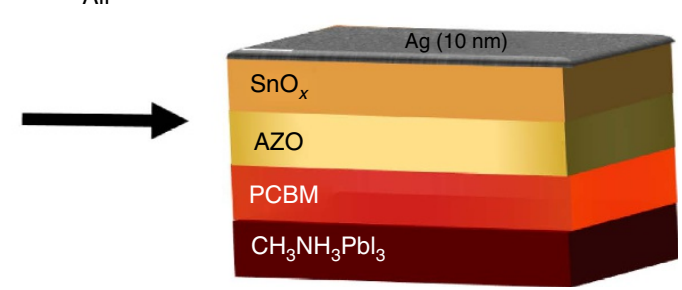

Figure 3 | Unravelling the ageing mechanism in air. XRD spectrum of a perovskite cell based on AZO as EEL after storage in air for 7 days (inset: magnified view of the (100) $\mathrm{Pbl}_{2}$ reflection for a fresh sample and one that has been stored in air for 7 days; spectra were vertically offset for clarity) (a). Photoemission spectra of the iodine $13 \mathrm{~d}_{5 / 2}$ peak for fresh and aged cells based on $\mathrm{AZO}, \mathrm{SnO}_{x}$ and bilayered $\mathrm{AZO} / \mathrm{SnO} \mathrm{O}_{x} \mathrm{EELs}$, respectively (b). The aged samples were stored in air for 2 days. Corresponding plan-view SEM images of the $10 \mathrm{~nm} \mathrm{Ag}$ layer in case of the AZO sample (c,d), and for the AZO/SnO ${ }_{x}$ sample (e). Schemes of ageing for the $\mathrm{AZO}$ and the $\mathrm{AZO} / \mathrm{SnO}_{x}$ samples $(\mathbf{f}, \mathbf{g})$.

As a result, decomposition of the $\mathrm{MAPbI}_{3}$ perovskite occurs and yields iodine-based products that migrate to the surface. In the case of $\mathrm{AZO} / \mathrm{SnO}_{x}$, substantially better moisture barrier properties prevent the penetration of water. The claim of superior moisture barrier properties of the bilayered $\mathrm{AZO} / \mathrm{SnO}_{x}$ EEL compared with that of the AZO NP layer is also supported by Ca-corrosion tests (Supplementary Fig. 6).

It has to be noted that the detrimental effects of ambient air could in principle be avoided by using a proper encapsulation of the entire solar cell. However, the out-diffusion of decomposition products from the perovskite and the degradation of the metal electrode (Fig. 3c,d) may impose a more fundamental issue. This is even more important, as earlier reports evidenced the thermally activated decomposition of the $\mathrm{MAPbI}_{3}$ perovskite even under inert atmosphere ${ }^{7}$.

Stability at elevated temperatures. To study the long-term stability of our solar cells under inert conditions, we have stored a set of devices in a glove box under $\mathrm{N}_{2}$ atmosphere. All the devices were placed on a hot plate at $60^{\circ} \mathrm{C}$ and their characteristics were measured repeatedly. Most remarkably, the characteristics of devices based on AZO EELs degraded on a timescale of $100 \mathrm{~h}$ (Fig. 4). Although the $J_{\mathrm{sc}}$ and $V_{\mathrm{oc}}$ of these devices remain 
a

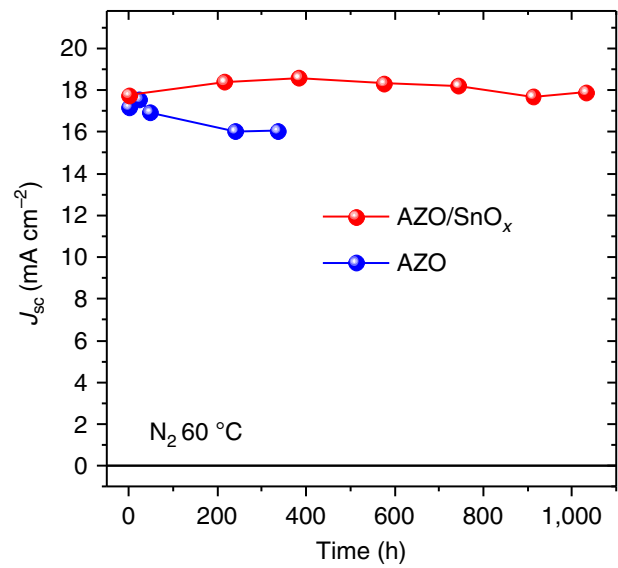

C

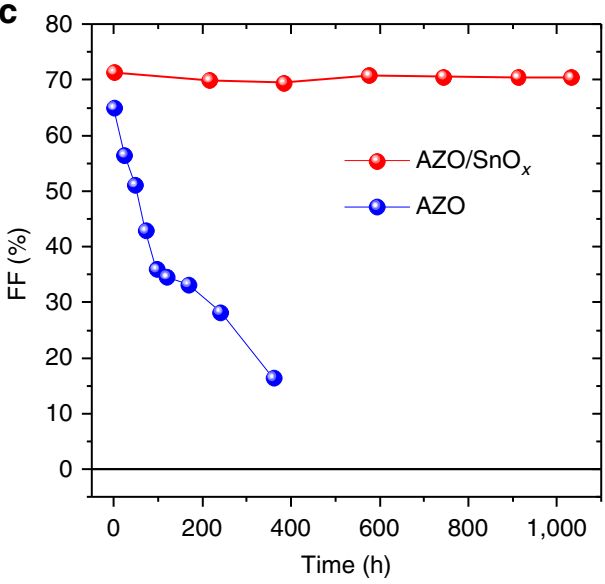

b

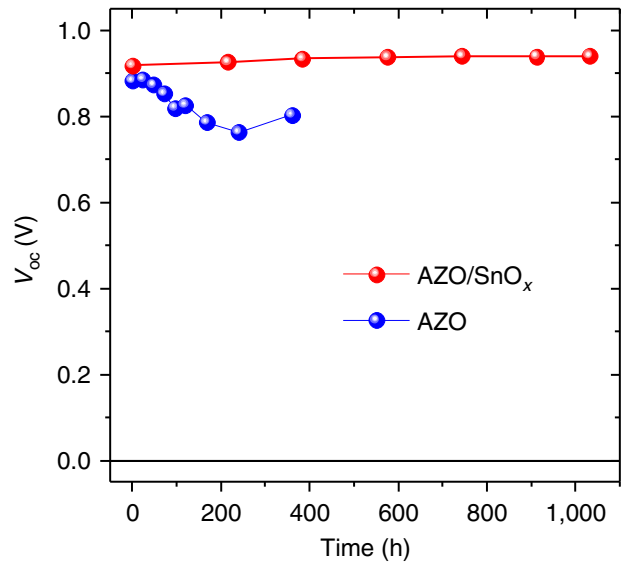

d

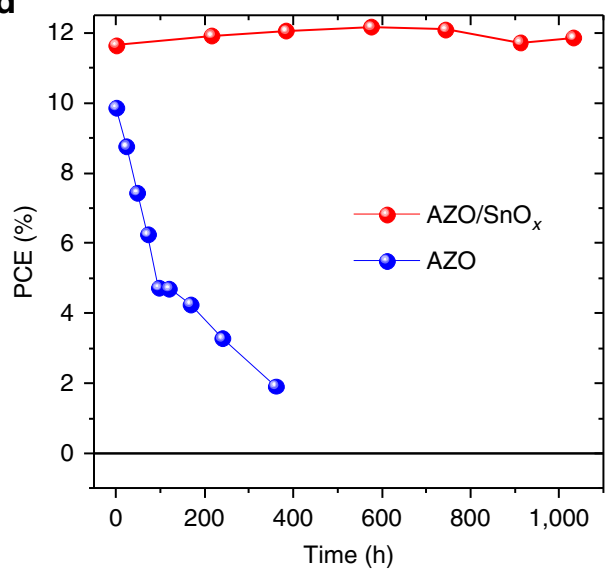

Figure 4 | Stability upon storage at $60^{\circ} \mathbf{C}$ in nitrogen atmosphere. Characteristics of inverted perovskite cells with varied cathode electron-extraction assemblies $\left(\mathrm{AZO} / \mathrm{SnO}_{x} / \mathrm{Ag}, \mathrm{AZO} / \mathrm{Ag}\right.$ ) versus time. $J_{\mathrm{sc}}(\mathbf{a}), \mathrm{V}_{\mathrm{oc}}(\mathbf{b}), \mathrm{FF}(\mathbf{c})$ and PCE $(\mathbf{d})$. In this set of samples, the nominal thickness of the ALD SnO ${ }_{x}$ layer was $20 \mathrm{~nm}$ and that of the $\mathrm{Ag}$ electrode was $100 \mathrm{~nm}$. The characteristics have been determined from the $J / V$ measurements in reverse direction (Supplementary Fig. 7).

fairly stable, the decay of the FF results in the severe drop of PCE to $\sim 2 \%$ after $360 \mathrm{~h}$. The degradation motif in the AZO-based cells is essentially different from that found in ambient air, where all cell characteristics decayed within a day (Fig. 2). Again in striking contrast, the characteristics of devices based on the bilayered $\mathrm{AZO} / \mathrm{SnO}_{x}$ EEL remain almost unchanged even after $1,032 \mathrm{~h}$. Note that the hysteresis did not increase in the course of ageing.

To unravel the mechanisms behind these results, we have again performed XRD and XPS studies of the respective device structures. After heating the device stacks for 6 days under inert atmosphere, the XRD spectrum of the AZO samples reveals a significant signal due to $\mathrm{PbI}_{2}$ that is absent in pristine stacks (Fig. 5a). The formation of $\mathrm{PbI}_{2}$ indicates the thermally activated decomposition of the perovskite upon heating, in agreement with earlier reports ${ }^{7}$. Opposed to that, the devices based on the bilayered $\mathrm{AZO} / \mathrm{SnO}_{x}$ EEL do not show any signature of a $\mathrm{PbI}_{2}$ phase after identical ageing conditions (Fig. 5b).

The formation of $\mathrm{PbI}_{2}$ in the AZO samples is in contrast to the degradation experiments in air, where no $\mathrm{PbI}_{2}$ phase in the AZO samples was found in XRD (Fig. 3a). At the same time, the $\mathrm{I} \mathrm{d}_{5 / 2}$ XPS spectra (Fig. 5c) do not reveal any significant increase of the amount of iodine at the surface of any of the samples aged in $\mathrm{N}_{2}$. Please note that the noise in the XPS spectra indicates that the concentration of $\mathrm{I}_{3 / 2}$ at the surface of all the samples is close to the detection limit and variations between the samples are within the measurement error. A plan-view SEM image of the aged AZO samples does not reveal any degradation of the Ag electrode, in contrast to the experiment where the ageing took place in air (Fig. 3c,d). Thus, we conclude that in the case of AZO, volatile decomposition products of the $\mathrm{MAPbI}_{3}$ perovskite evaporate from the cell stack without degradation of the Ag electrode. In air, other than in $\mathrm{N}_{2}$, the presence of water would promote the corrosion of $\mathrm{Ag}$ because of $\mathrm{CH}_{3} \mathrm{NH}_{3} \mathrm{I}$ and other halide compounds ${ }^{10}$. However, the loss of $\mathrm{CH}_{3} \mathrm{NH}_{3} \mathrm{I}$ etc. drives the further decomposition of the perovskite.

\section{Discussion}

Taken together, the device characteristics (Fig. 4) and the XRD and XPS spectroscopy point to a scheme of degradation as shown in Fig. 5e,f. Under inert atmosphere, the thermally activated degradation of the perovskite leads to decomposition products that can easily diffuse through the PCBM/AZO/Ag layers on top of the perovskite. The less volatile $\mathrm{PbI}_{2}$ phase remains in the active layer leading to a substantial decay of the FF of the corresponding devices (Fig. 4c). Moreover, it has been shown earlier that a change in film stoichiometry $(\mathrm{Pb}$ poor to $\mathrm{Pb}$ rich) may substantially alter the electronic properties of the perovskite and especially the position of the conduction and valence band with respect to the vacuum level ${ }^{30}$. Towards 
a

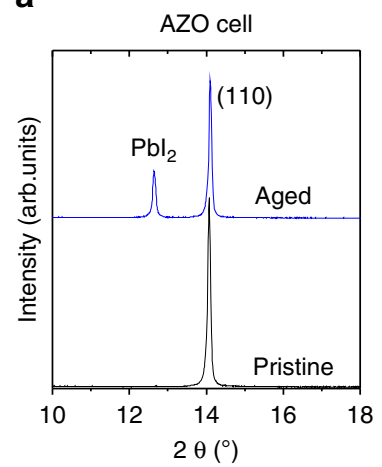

d

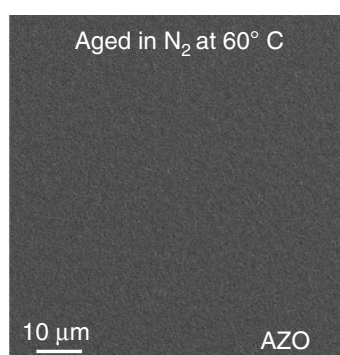

b

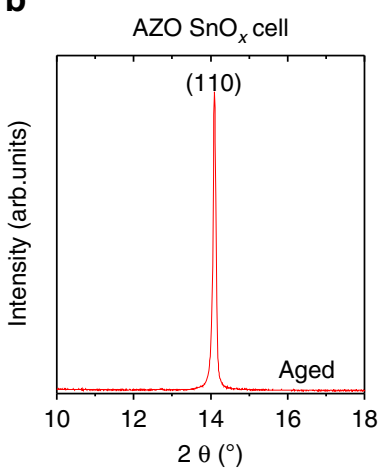

e

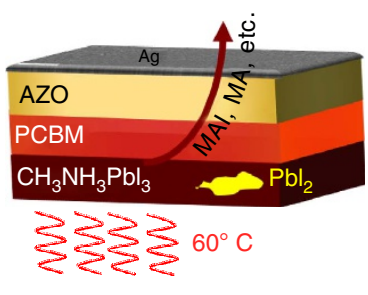

c

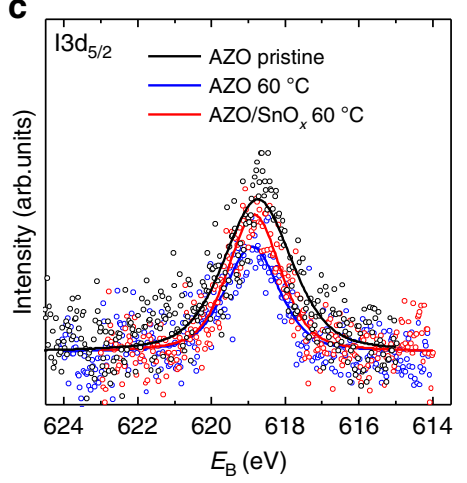

f

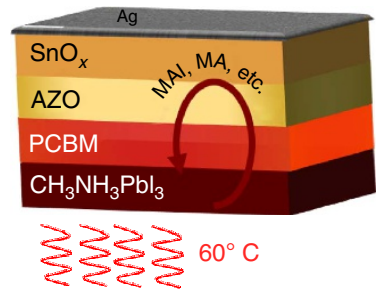

Figure 5 | Why impermeable $\mathbf{A Z O}_{\mathbf{S n O}} \mathbf{E E L s}_{\mathbf{x}}$ afford stable devices. XRD spectra of a perovskite cell based on $\mathrm{AZO}$ and $\mathrm{AZO} / \mathrm{SnO} \mathrm{O}_{x}$ as $\mathrm{EEL}$ before and after heating in nitrogen for 6 days. $(\mathbf{a}, \mathbf{b})$ Photoemission spectra of the iodine $13 \mathrm{~d}_{5 / 2}$ peak for cells based on $\mathrm{AZO}$ and bilayered $\mathrm{AZO} / \mathrm{SnO} \mathrm{O}_{x} \mathrm{EELs}$, respectively. (c) As a reference, an AZO sample stored in $\mathrm{N}_{2}$ ambient has been used. The other measurements are for samples aged at $60^{\circ} \mathrm{C}$ in nitrogen for 6 days. The open symbols represent the raw measurement data, whereas the solid lines are a result of a peak fit. The colours are defined in the figure legend. Note that the top $\mathrm{Ag}$ electrode was chosen to be $10 \mathrm{~nm}$. Corresponding plan-view SEM image of the $10 \mathrm{~nm} \mathrm{Ag}$ layer in case of the aged AZO sample. (d) Schemes of ageing and decomposition of the perovskite in case of the $\mathrm{AZO}(\mathbf{e})$ and the AZO/SnO $\mathrm{S}_{x}$ samples (f). The yellow spot in the scheme of the AZO sample symbolizes the formation of $\mathrm{Pbl}_{2}$.

increasingly $\mathrm{Pb}$-rich conditions, the ionization energy of the perovskite has been shown to increase significantly to values in excess of $6 \mathrm{eV}$ that would render the extraction of holes more and more challenging.

Opposed to the case of the AZO cells, the $\mathrm{AZO} / \mathrm{SnO}_{x}$-based cells do not show any formation of $\mathrm{PbI}_{2}$ under otherwise identical conditions. We therefore conclude that the decomposition products are contained inside the cell stack because of the dense $\mathrm{SnO}_{x}$ layer forming a permeation barrier. Thereby, the equilibrium of the decomposition/formation reaction would be shifted to stabilize the perovskite. Thus, the decomposition of the $\mathrm{MAPbI}_{3}$ obviously is significantly slowed down, and the cells do not show significant degradation even after heating for $>1,000 \mathrm{~h}$ (Fig. 4). It has to be noted that it is favourable to position the permeation barrier between the perovskite and the Ag electrode, as thereby the known long-term corrosive effect of residual halide components on the $\mathrm{Ag}$ electrode $^{31}$ and on the interface EEL/Ag can be efficiently suppressed. In further work, this concept could even be extended to a setup, in which the perovskite layer is sandwiched between diffusion barriers at the cathode and anode side, in case permeable substrates like polymer foils are used. For example, there are reports on using ALD to prepare $\mathrm{V}_{2} \mathrm{O}_{5}$ layers ${ }^{32}$. $\mathrm{V}_{2} \mathrm{O}_{5}$ is a high work function transition metal oxide that has successfully been used for hole extraction in organic and perovskite solar cells ${ }^{33-36}$. ALD prepared $\mathrm{V}_{2} \mathrm{O}_{5}$ would presumably provide similar gas permeation barrier properties as those shown for the $\mathrm{SnO}_{x}$. Thereby, the intrinsic decomposition of the perovskite active layers would be substantially delayed or even prevented as volatile decomposition products would be hindered from evaporating.
Aside from the so-called mono-stress conditions like elevated temperature, multi-stressing can be considered. To this end, we conducted set of long-term light soaking tests at $60^{\circ} \mathrm{C}$ under $\mathrm{N}_{2}$, in which the devices were simultaneously illuminated with a white light-emitting diode (LED) to achieve the same $J_{\text {sc }}$ as upon AM1.5G solar irradiation (for details see the Methods section). The results of this multi-stress experiment are shown in the Supplementary Fig. 8. Briefly, again the $\mathrm{AZO} / \mathrm{SnO}_{x}$ cells are substantially more stable than the AZO cells under concomitant heat and illumination. This difference is in part because of the suppressed decomposition of the perovskite owing to heat in case of the $\mathrm{AZO} / \mathrm{SnO}_{x}$ (in agreement with the discussion of Fig. 4). However, there is a clear degradation even of the $\mathrm{AZO} / \mathrm{SnO}_{x}$ samples where the PCE decays to roughly $60 \%$ of its initial value after $300 \mathrm{~h}$. This is in contrast to the case where only thermal stress has been used. Earlier work has unravelled the photo-induced degradation of solar cells based on $\mathrm{MAPbI}_{3}$. Whereas Misra et al. ${ }^{37}$ identified a photoactivated decomposition mechanism, more recently Nie et al. ${ }^{38}$ reported that continuous illumination of $\mathrm{MAPbI}_{3}$ caused the formation of trap states that spoiled the solar cell performance, especially $J_{\mathrm{sc}}$. Although our sealing approach based on the impermeable $\mathrm{AZO} / \mathrm{SnO}_{x}$ electron extraction layer has been shown to efficiently suppress the decomposition of the perovskite, it cannot suppress the formation of trap states in the perovskite. Similar to the report of Nie et al. ${ }^{38}$ the cell characteristics recover after stressing, if the cells are kept in darkness (Supplementary Fig. 8f). This is why we conclude that photo-induced trap-state formation occurs in our cells. However, there have been recent reports confirming that photo-induced degradation is not a general problem of organolead halide perovskites, and some optimized mixed cation/ 
mixed halide perovskite active materials were shown to be far less prone to light soaking degradation ${ }^{24,39}$. Heat was found to be a dominating source of degradation in these mixed cation perovskite cells ${ }^{40}$. Note that our inverted device structure based on the impermeable $\mathrm{AZO} / \mathrm{SnO}_{x}$ electron extraction layer is generally applicable and can also accommodate these nextgeneration perovskite photoactive systems with enhanced stability and efficiency ${ }^{41}$.

In summary, we have shown an ultra-stable inverted cell architecture, where a bilayered $\mathrm{AZO} / \mathrm{SnO}_{x} \mathrm{EEL}$ has been demonstrated to afford perovskite solar cells with a remarkable resilience. The $20 \mathrm{~nm}$ thin $\mathrm{ALD}$ grown $\mathrm{SnO}_{x}$ has been evidenced to form an outstandingly dense gas permeation barrier that effectively hinders the ingress of moisture towards the perovskite and-more importantly-it prevents the egress of decomposition products like $\mathrm{CH}_{3} \mathrm{NH}_{3} \mathrm{I}$, HI out of the perovskite.

Although the efficiency of cells based on $\mathrm{LiF} / \mathrm{Al}$ or AZO degraded to $<50 \%$ of the initial value within less than a day in ambient air, devices based on $\mathrm{AZO} / \mathrm{SnO}_{x}$ showed superior stability of their solar cell characteristics even on a timescale of $>350 \mathrm{~h}$. Under inert atmosphere, AZO-based devices degraded to roughly $50 \%$ of its initial efficiency within $100 \mathrm{~h}$ because of thermally induced decomposition of the perovskite. On the contrary, the suppressed out-diffusion of decomposition products because of the diffusion-barrier properties of the $\mathrm{AZO} / \mathrm{SnO}_{x}$ EEL has been shown to significantly slow down the thermally activated decomposition of the perovskite. This afforded cells that did not change even after $>1,000 \mathrm{~h}$. The concept of impermeable EELs is generally applicable and therefore is expected to provide an avenue to achieve a substantially improved device lifetime of solar cells based on organometal halide perovskites even beyond $\mathrm{MAPbI}_{3}$.

\section{Methods}

Material synthesis and device preparation. The inverted perovskite solar cells studied in this work are based on the following layer sequence: glass/ITO/ PEDOT:PSS/perovskite/PCBM/EEL/Ag (see Fig. 1a). The PEDOT layer (AI4083) has been spin coated in ambient air and dried on a hot plate at $120^{\circ} \mathrm{C}$ for $20 \mathrm{~min}$ in air and for another $20 \mathrm{~min}$ in a glove box under $\mathrm{N}_{2}$ atmosphere. The perovskite layer has been spin coated under inert atmosphere from a commercially available precursor solution (Ossila) and the resulting layers were thermally annealed at $100^{\circ} \mathrm{C}$ for $80 \mathrm{~min}$. The typical layer thickness was $180 \mathrm{~nm}$.

On top of the perovskite, PCBM (American Dye Sorce Inc., ADS61BFA) was spin coated from a solution in chlorobenzene (concentration: $100 \mathrm{mg} \mathrm{ml}^{-1}$ ). AZO has been deposited from a commercial nanoparticle dispersion (2.5 wt\% in isopropanol, Prod. No. 8045, Nanograde AG, Switzerland). Tin oxide has been prepared by atomic layer deposition in a Beneq TFS 200 system (base pressure $1.5 \mathrm{mbar}$ ). As precursors, tetrakis(dimethylamino)tin(IV) (TDMASn), kept at $45^{\circ} \mathrm{C}$ and water, kept at room temperature, were used. At a substrate temperature of $80^{\circ} \mathrm{C}$ the growth rate per cycle was $1.1 \AA$. Further details on the materials properties of the $\mathrm{SnO}_{\mathrm{x}}$ can be found in our earlier work ${ }^{23,27}$. Ag (100 or $\left.10 \mathrm{~nm}\right)$ layers were thermally evaporated in high vacuum $\left(10^{-7} \mathrm{mbar}\right)$. In some reference devices, $\mathrm{LiF}(1 \mathrm{~nm})$ and $\mathrm{Al}(100 \mathrm{~nm})$ have been thermally evaporated as cathode.

Materials and device characterization. The electrical conductivity was measured with the Van der Pauw method.

The SEM studies were conducted using Neon 40 (Zeiss).

XRD was measured using a monochromatic $\mathrm{Cu}-\mathrm{K} \alpha_{1,2}$ source (Philips X'Pert Pro MPD). XPS has been performed using a non-monochromatic $\mathrm{Mg} \mathrm{K} \mathrm{X}_{\alpha} \mathrm{X}$-ray source (VG) and a Phoibos 100 (Specs) electron analyser.

Rutherford backscattering was measured at RUBION (University of Bochum, Germany) using a dynamitron tandem accelerator with $2 \mathrm{MeV}^{4} \mathrm{He}^{+}$beam (beam current of 20-40 nA). A silicon surface barrier detector was placed at an angle of $160^{\circ}$ with respect to the beam axis. The solid angle of the detector was 1.91 msrad.

The solar cells were characterized in ambient air without encapsulation using a Keithley 2400-C source meter and a solar simulator (300 W Newport, model 91160, AM1.5G, $100 \mathrm{~mW} \mathrm{~cm}^{-2}$ ). We used a commercial Si reference cell (Rera Systems) that is IEC 60904-9 compliant and certified. Short circuit current was obtained from the EQE data. EQE has been measured with a home-built tunable light source, calibrated with a power meter (Thorlabs) The spectral mismatch factor was 1.163
Some of the devices have been kept in a glovebox under $\mathrm{N}_{2}$ atmosphere on a hot plate at $60^{\circ} \mathrm{C}$. The $\mathrm{J} / \mathrm{V}$ characteristics were measured in forward and reverse direction at a scanning speed of $500 \mathrm{mV} \mathrm{s}^{-1}$. Cells were measured using a mask with round aperture of $1.5 \mathrm{~mm}$ diameter. Stabilized current was measured in the same $J / V$ setup using a voltage source.

Long-term light soaking tests at $60^{\circ} \mathrm{C}$ under $\mathrm{N}_{2}$ were conducted by simultaneously illuminating the cells with a white LED to achieve the same $J_{\mathrm{sc}}$ as upon AM1.5G solar irradiation. The LED spectrum is shown in the Supplementary Fig. 8e. The cells were mounted in a home-built oven inside a glove box. They were kept in short circuit conditions during the ageing experiment

Data availability. The data that support the findings of this study are available from the corresponding author on request.

\section{References}

1. Graetzel, M. The light and shade of perovskite solar cells. Nat. Mater. 13, 838-842 (2014).

2. Yang, W. S. et al. High-performance photovoltaic perovskite layers fabricated through intramolecular exchange. Science 348, 1234-1237 (2015).

3. Tiep, N. H., Ku, Z. \& Fan, H. J. Recent advances in improving the stability of perovskite solar cells. Adv. Energy Mater. 6, 1501420 (2016).

4. Leijtens, T. et al. Stability of metal halide perovskite solar cells. Adv. Energy Mater. 5, 1500963 (2015).

5. Berhe, T. A. et al. Organometal halide perovskite solar cells: degradation and stability. Energ. Environ. Sci. 9, 323-356 (2016).

6. Niu, G. et al. Study on the stability of $\mathrm{CH}_{3} \mathrm{NH}_{3} \mathrm{PbI}_{3}$ films and the effect of postmodification by aluminum oxide in all-solid-state hybrid solar cells.

J. Mater. Chem. A 2, 705-710 (2014).

7. Conings, B. et al. Intrinsic thermal instability of methylammonium lead trihalide perovskite. Adv. Energy Mater. 5, 1500477 (2015).

8. Divitini, G. et al. In situ observation of heat-induced degradation of perovskite solar cells. Nat. Energy 1, 15012 (2016)

9. Kim, A. et al. Fully solution-processed transparent electrodes based on silver nanowire composites for perovskite solar cells. Nanoscale 8, 6308-6316 (2016).

10. Kato, Y. et al. Silver iodide formation in methyl ammonium lead iodide perovskite solar cells with silver top electrodes. Adv. Mat. Interfaces 2, 1500195 (2015).

11. Nie, W. et al. High-efficiency solution-processed perovskite solar cells with millimeter-scale grains. Science 347, 522-525 (2015).

12. Heo, J. H., Han, H. J., Kim, D., Ahn, T. K. \& Im, S. H. Hysteresis-less inverted $\mathrm{CH}_{3} \mathrm{NH}_{3} \mathrm{PbI}_{3}$ planar perovskite hybrid solar cells with $18.1 \%$ power conversion efficiency. Energ. Environ. Sci. 8, 1602-1608 (2015).

13. Jeng, J.-Y. et al. $\mathrm{CH}_{3} \mathrm{NH}_{3} \mathrm{PbI}_{3}$ perovskite/fullerene planar-heterojunction hybrid solar cells. Adv. Mater. 25, 3727-3732 (2013).

14. You, J. et al. Improved air stability of perovskite solar cells via solutionprocessed metal oxide transport layers. Nat. Nano. 11, 75-81 (2016).

15. Chen, W. et al. Efficient and stable large-area perovskite solar cells with inorganic charge extraction layers. Science 350, 944-948 (2015).

16. Hirvikorpi, T., Vähä-Nissi, M., Nikkola, J., Harlin, A. \& Karppinen, M. Thin Al2O3 barrier coatings onto temperature-sensitive packaging materials by atomic layer deposition. Surf. Coat. Technol. 205, 5088-5092 (2011).

17. Kaltenbrunner, M. et al. Flexible high power-per-weight perovskite solar cells with chromium oxide-metal contacts for improved stability in air. Nat. Mater. 14, 1032-1039 (2015).

18. Guerrero, A. et al. Interfacial degradation of planar lead halide perovskite solar cells. ACS Nano 10, 218-224 (2016).

19. Bush, K. A. et al. Thermal and environmental stability of semi-transparent perovskite solar cells for tandems enabled by a solution-processed nanoparticle buffer layer and sputtered ITO electrode. Adv. Mater. 28, 3937-3943 (2016).

20. Yang, J., Siempelkamp, B. D., Mosconi, E., De Angelis, F. \& Kelly, T. L. Origin of the thermal instability in $\mathrm{CH}_{3} \mathrm{NH}_{3} \mathrm{PbI}_{3}$ thin films deposited on $\mathrm{ZnO}$. Chem. Mater. 27, 4229-4236 (2015).

21. Trost, S. et al. Overcoming the 'light-soaking' issue in inverted organic solar cells by the use of $\mathrm{Al}: \mathrm{ZnO}$ electron extraction layers. Adv. Energy Mater. 3, 1437-1444 (2013).

22. Kim, I. S. Martinson ABF. Stabilizing hybrid perovskites against moisture and temperature via non-hydrolytic atomic layer deposited overlayers. J. Mater. Chem. A 3, 20092-20096 (2015).

23. Behrendt, A. et al. Highly robust transparent and conductive gas diffusion barriers based on tin oxide. Adv. Mater. 27, 5961-5967 (2015).

24. McMeekin, D. P. et al. A mixed-cation lead mixed-halide perovskite absorber for tandem solar cells. Science 351, 151-155 (2016).

25. Deng, Y., Dong, Q., Bi, C., Yuan, Y. \& Huang, J. Air-stable, efficient mixed-cation perovskite solar cells with $\mathrm{Cu}$ electrode by scalable fabrication of active layer. Adv. Energy Mater. 6, 1600372 (2016). 
26. Han, Y. et al. Degradation observations of encapsulated planar $\mathrm{CH}_{3} \mathrm{NH}_{3} \mathrm{PbI}_{3}$ perovskite solar cells at high temperatures and humidity. J. Mater. Chem. A 3, 8139-8147 (2015)

27. Trost, S. et al. Tin oxide ( $\mathrm{SnOx}$ ) as universal 'light-soaking' free electron extraction material for organic solar cells. Adv. Energy Mater. 5, 1500277 (2015).

28. Knez, M., Niesch, K. \& Niinisto, L. Synthesis and surface engineering of complex nanostructures by atomic layer deposition. Adv. Mater. 19, 3425-3438 (2007).

29. Wilson, C. A., Grubbs, R. K. \& George, S. M. Nucleation and growth during $\mathrm{Al} 2 \mathrm{O} 3$ atomic layer deposition on polymers. Chem. Mater. 17, 5625-5634 (2005).

30. Emara, J. et al. Impact of film stoichiometry on the ionization energy and electronic structure of $\mathrm{CH}_{3} \mathrm{NH}_{3} \mathrm{PbI}_{3}$ perovskites. Adv. Mater. 28, 553-559 (2016).

31. Bryant, D. et al. A transparent conductive adhesive laminate electrode for highefficiency organic-inorganic lead halide perovskite solar cells. Adv. Mater. 26, 7499-7504 (2014).

32. Blanquart, T. et al. Atomic layer deposition and characterization of vanadium oxide thin films. RSC Adv. 3, 1179-1185 (2013).

33. Meyer, J., Zilberberg, K., Riedl, T. \& Kahn, A. Electronic structure of vanadium pentoxide: an efficient hole injector for organic electronic materials. J. Appl. Phys. 110, 033710 (2011).

34. Zilberberg, K., Trost, S., Schmidt, H. \& Riedl, T. Solution processed vanadium pentoxide as charge extraction layer for organic solar cells. Adv. Energy Mater. 1, 377-381 (2011).

35. Meyer, J. et al. Transition metal oxides for organic electronics: energetics, device physics and applications. Adv. Mater. 24, 5408-5427 (2012).

36. Back, H. et al. Achieving long-term stable perovskite solar cells via ion neutralization. Energ. Environ. Sci. 9, 1258-1263 (2016).

37. Misra, R. K. et al. Temperature- and component-dependent degradation of perovskite photovoltaic materials under concentrated sunlight. J. Phys. Chem. Lett. 6, 326-330 (2015).

38. Nie, W. et al. Light-activated photocurrent degradation and self-healing in perovskite solar cells. Nat. Commun. 7, 11574 (2016).

39. Saliba, M. et al. Incorporation of rubidium cations into perovskite solar cells improves photovoltaic performance. Science 354, 206-209 (2016).

40. Domanski, K. et al. Not all that glitters is gold: metal-migrationinduced degradation in perovskite solar cells. ACS Nano 10, 6306-6314 (2016).

41. Liu, J. et al. High-quality mixed-organic-cation perovskites from a phase-pure non-stoichiometric intermediate $(\mathrm{FAI})_{1-x}-\mathrm{PbI}_{2}$ for solar cells. Adv. Mater. 27, 4918-4923 (2015).

\section{Acknowledgements}

We acknowledge the German Federal Ministry for Education and Research (Grant No. 03EK3529E) and the Deutsche Forschungsgemeinschaft (DFG) (Grants: RI1551/4-2) for financial support. We acknowledge the Ministry of Science of the state of NRW for funding within the PeroBOOST (EFRE) project. The research leading to these results has received partial funding from the European Unions 7th Framework Programme under Grant Agreement no. 604148 (MUJULIMA).

\section{Author contributions}

T.R. and K.O.B. conceived and designed the experiments. S.O. and K.M. contributed the XPS and plan-view SEM analysis. N.P. did the XRD study. D.R. contributed the Rutherford backscattering (RBS) analysis. K.O.B., T.B., T.H. and J.Z. performed the experimental work on the perovskite cell devices. L.H. and T.G. contributed the tin oxide ALD layers as well as electrical and permeation barrier studies. R.H. did the cross-section SEM measurements. M.F.O. and N.A.L. provided the AZO dispersions along with the expertise of their processing. All authors discussed the results and were involved in the writing.

\section{Additional information}

Supplementary Information accompanies this paper at http://www.nature.com/ naturecommunications

Competing financial interests: The authors declare no competing financial interests.

Reprints and permission information is available online at http://npg.nature.com/ reprintsandpermissions/

How to cite this article: Brinkmann, K. O. et al. Suppressed decomposition of organometal halide perovskites by impermeable electron-extraction layers in inverted solar cells. Nat. Commun. 8, 13938 doi: 10.1038/ncomms13938 (2017).

Publisher's note: Springer Nature remains neutral with regard to jurisdictional claims in published maps and institutional affiliations.

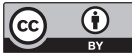

This work is licensed under a Creative Commons Attribution 4.0 International License. The images or other third party material in this article are included in the article's Creative Commons license, unless indicated otherwise in the credit line; if the material is not included under the Creative Commons license, users will need to obtain permission from the license holder to reproduce the material. To view a copy of this license, visit http://creativecommons.org/licenses/by/4.0/

(C) The Author(s) 2017 